\title{
Parameters Describing the Pulse Wave
}

\section{KORPAS, J. HÁLEK, L. DOLEŽAL}

\author{
Department of Medical Biophysics, Faculty of Medicine, Palacký University Olomouc, Czech \\ Republic
}

Received December 8, 2007

Accepted July 2, 2008

On-line July 25, 2008

\section{Summary}

Pulse wave analysis permits non-invasive assessment of arterial elasticity indices. The contour varies in different parts of the circulation. It depends on physiological or pathophysiological conditions of the organism. The pathological events like arteriosclerosis or diabetes have a primary effect to the artery elasticity. Hypertension or some heart diseases also influence the pulse wave velocity and resulted in earlier wave reflections. There are several methods of pulse wave measurements based on different principles and depending on the type of measured pulse wave. The evaluation parameters can be assessed from the time domain, derivations, velocity or frequency domain. The main aim of this review article is to offer a recent overview of pulse wave measurement parameters and main results obtained. The principles of pulse wave measurement and current experience in clinical practice are shortly discussed too.

\section{Key words}

Arterial elasticity - Blood flow - Measurement techniques • Plethysmography • Pulse wave

\section{Corresponding author}

D. Korpas, Faculty of Medicine, Department of Medical Biophysics, Hnevotinska 3, 77515 Olomouc, Czech Republic. E-mail: david.korpas@seznam.cz

\section{Introduction}

The pulse wave (PW) is a complex physiological phenomenon observed and detected in blood circulation. In the course of heart systole a certain amount of blood is ejected and it is moved into the arteries because of transformation between kinetic and potential energy of each segment of ejected blood. On each artery or venous section affected by a pulse wave, three coherent phenomena can be observed: blood flow (flow pulse), the increase of blood pressure (pressure pulse) and extension of transverse profile (profile or volume pulse). Several invasive methods are available to detect the PW. The PW contour varies in different parts of the circulation. It depends on physiological or pathophysiological conditions of the organism. The heart rate, the body height and the age (Filipovsky et al. 2000), as well as BMI or body fat (Wykretowicz et al. 2007) belong to important physiological phenomena. The pathological events like arteriosclerosis or diabetes have a great primary effect on the arterial elasticity (Nichols 2005). Hypertension or some heart diseases influence the PW velocity.

Increased arterial stiffness is an important marker of arteriosclerosis, which is the main cause of cardiovascular mortality in developed countries. During the life, the arterial wall constantly loses elasticity and becomes rigid (Kelly et al. 1989, Nichols and O'Rourke 1998, Vaitkevicius et al. 1993). Hypertension and other diseases like diabetes mellitus accelerate this process (Brooks et al. 1999). The increase in artery wall stiffness is noticeable from the beginning of the arteriosclerosis process, before anatomical changes and clinical manifestations are observed. Some methods for arterial stiffness measurement are based on the measurement of pulse wave velocity and analysis of pulse wave curve form (Oliva and Roztočil 1982, Savage et al. 2002, Wilkinson et al. 2000). 


\section{Pulse wave physiology}

The physics of blood circulation in arteries can be demonstrated on the model mechanical system. This system is composed of a piston and elastic hose, which is filled with fluid and terminated by wave resistance. The piston (model of heart) injects a certain volume of fluid (blood) into the hose (model of arteries). If we have a rigid hose, the whole volume of fluid would move, but if we have an elastic-walled-hose, only the fluid at the beginning of the hose moves. The pressure then increases, which causes local widening of the hose and local accumulation of the fluid. Tension of the widened elastic wall causes contraction and pushes the fluid to the next part of the hose. The hose widens there again and the pressure increases. The process continues along the whole length of the hose. The process, which we have just described, runs continuously as a propagating pulse wave along the hose. In every region where the pulse wave runs, three related effects can be observed: liquid flow (flow wave), a pressure change (pressure wave), and a cross-section change (volume wave).

The forward wave spreads to the periphery, where it does not dissipate, but is reflected back to central vascular system. This retrograde propagation causes an increase of blood pressure again. Standing waves arise. The resulting pulse waveform is determined by phase sum of forward and reflected waves. The time differences of reflected pulse wave in each part of circulation are responsible for differences of central and peripheral pulse wave. The pressure in ascending aorta decreases after reaching the maximum and there is a typical incision at the end of diastole. This is caused by short regurgitation at the end of systole. It closes the semilunar valves and the systole is finished. These shapes of pressure pulse are typical for the arteries close to the heart, e.g. subclavian artery or carotid artery.

In distal arteries, the pressure changes are different from those in the aorta. The pulse pressure increases there and there is no incision because of attenuation of higher frequencies. Therefore the curves are more rounded. The peripheral pulse wave has two parts: primary and dicrotic. The beginning flash increase of the pressure is caused by slower drain to capillaries then inflow from the large arteries. Subsequent pressure decrease is caused by lower inflow from the heart then by drain to capillaries. The pulse shape is influenced by the dicrotic wave. This is caused by standing waves between ascending aorta and the distal reflect part at the limb. This standing wave creates maxima and minima, which influences the dicrotic part of the pulse curve. The amplitude of these standing waves is clearer at the distal arteries and the shape of the flow wave differs from the pressure wave and volume wave. Within the superposition of the opposite wave the pressures and volumes are added but the flows are subtracted. The above mentioned should be taken into the consideration to evaluate the pulse wave properly.

\section{Diagnostic techniques}

There are several methods of pulse wave measurements based on different principles and depending on what type of the pulse wave should be measured. We will describe the non-invasive methods only. In this case, the pulse wave spreading in the surface arteries impact the sensor either directly or implicitly modulate an energetic impulse transmitted to the body. According to the division mentioned above, we can obtain three following waves.

\section{Pressure pulse wave}

The pressure pulse wave changes its shape while moves to the distal arteries. These changes are thought to be largely due to the pulse wave reflection and the tapering. The pressure pulse wave has similarities with volume pulse wave, meaning the similar changes occurring in conjunction with vascular diseases, such as damping and a loss of pulsatility because of absorbing the higher frequencies of artery wall movement because of its rigidity. Very famous method (Peňáz 1973, 1989) of noninvasive pressure pulse wave assessment uses fingerpressure cuff in feedback of photoplethysmography sensor to control the constant pressure. There are several commercial devices (Finapres, Ohmeda, Madison, USA) that can be used for this purpose.

\section{Volume pulse wave}

The easiest way is to use the classic brachial cuff surrounding the limb and attached to the pressure transducer. This technique is not very sensitive since the addition of the pulse waves of the all arteries under the cuff is also measured. But this still can be used in venous occlusion plethysmography for assessment of the blood volume flowed into the limb after occlusion or for measurement of pulse wave velocity between two cuffs. 
The most widespread method used is photoplethysmography. This technique is an optical measurement technique that is used to detect blood volume changes in the microvascular bed of tissue (Challoner 1979), and often used in clinical research (Blanc et al. 1993). This principle is also used in oxymeters or blood pressure measurement systems. A summary of the principles and applications of photoplethysmography were recently described by Allen (2007).

Sphygmography is a method for sensing the pulse due to probe, which is pressed to the suitable surface artery. Capacity, inductive or resistive probes can be used (Guyton and Hall 1996, Schurmann et al. 2001). The disadvantage is a small sensitivity of this devices and also abnormal compressing of the measured vessel. Using the elastic membrane as a probe can eliminate these disadvantages (Hálek and Korpas 2003, Kutal et al. 2003).

\section{Flow pulse wave}

Doppler ultrasound flowmeter is now widely used type for measurement of the flow. This can be applied from body surface and has a lot of advantages. Erythrocytes are the basic echo structures and frequencies from $2 \mathrm{MHz}$ to $10 \mathrm{MHz}$ are used. With the color Doppler techniques combined with pulsed emission (to determine the spatial flow location) we can measure the mean flow velocity in selected vessel. However, the flow pulse wave shape does not correlate with the volume and pressure waves. A negative part can also occur here during the regurgitation phase of blood flow.

Using the above mentioned methods we must concern about the pulse wave type we measure. There are also relationships between the central and peripheral pulse waves (Takazawa et al. 2007). Central pulse wave can be estimate using the transfer function (Chen et al. 1997, Hope et al. 2007).

\section{Evaluated parameters}

The following review should summarize the parameters used currently in the scientific literature. The variation coefficients are usually about five percent. Physiological changes of the variables influencing the pulse wave (heart frequency, low circulatory rhythms, etc.) should be always taken into consideration and the appropriate measurement protocol should be set.
Measurement in supine position after several minutes of rest and with synchronized breathing is recommended.

\section{Measurement on pulse wave in time domain}

The typical volume or pressure pulse wave shape of the peripheral (radial) artery is shown in Figure 1. Of course, there are no pathophysiological events like pulse bispheriens or wide pulse peak. There are two dicrotic waves. On the time course of pulse wave there are following proportions which can be measured: crest time (TP1), dicrotic wave time (TP2), total pulse duration (TPT), interwave time (IWT) measured in two thirds of systolic peak, second forward wave time (T2), systolic amplitude (AP1), dicrotic wave amplitude (AP2).

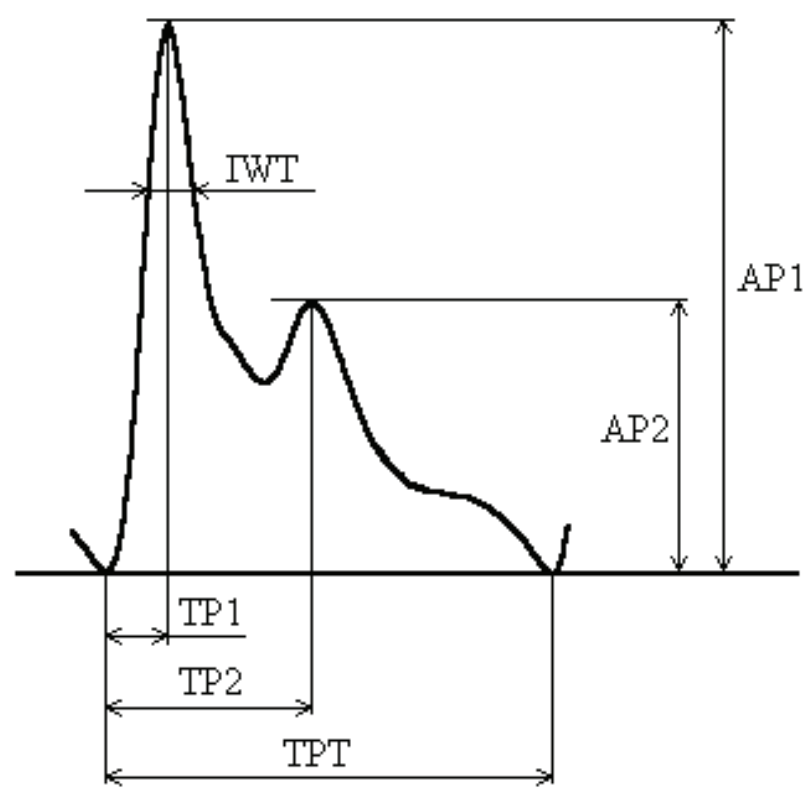

Fig. 1. Relevant proportions of volume or pressure pulse wave shape of the peripheral artery.

From the above mentioned proportions, the following parameters can be derived: relative crest time $(\mathrm{RCT})=\mathrm{TP} 1 / \mathrm{TPT}$, interwave distance $(\mathrm{IWD})=$ IWT/TPT, relative dicrotic wave amplitude (DWA) = AP2/AP1 (also called index of pressure wave reflection or reflection index (RI)) or relative dicrotic wave time $(\mathrm{DWT})=$ TP2/TPT, all these parameters are dimensionless. In practice the augmentation index is used for the expression of intraarterial pressure increase caused by reflected wave. The aortic wave curve is calculated from radial curve using the transfer function. Using the body height (h), the index of large artery stiffness (SI) $=\mathrm{h} / \mathrm{T} 2$ can be calculated. SI is related to PWV (pulse wave 
velocity) in large arteries and closely correlates with carotid-to-femoral PWV (Millasseau et al. 2002, 2003). Time delay parameter $(\mathrm{DT})=\mathrm{TP} 2-\mathrm{TP} 1$ is also used (Šimek et al. 2005) as arrival time of reflected waves at the central aorta when measuring the pressure waveform (Vlachopoulos et al. 2000, O'Rourke et al. 2001). The variation coefficients for SI and DT are about $2.42 \%$, for RI about $5.32 \%$ (Šimek et al. 2005)

Millasseau et al. (2003) also divide the whole pulse to systolic and diastolic part. The systolic component of the waveform arises mainly from a forward going pressure wave and the diastolic component from pressure waves reflected back along the aorta from small arteries mainly in the lower body. The time between the systolic and diastolic peaks is related to the transit time of the pressure waves from the root of the aorta to the site of reflection and back.

There are some other time-derived parameters like inclination time, elasticity index, descending time, propagation time (Oliva and Roztočil 1982), which are not used any more because of difficult evaluation.

\section{Measurement on pulse wave derivatives}

Another approach is the evaluation of derivations of the waveforms. This allows us the precise analysis of sudden changes in the waveform and time shifts. Figure 2 shows a real volume pulse waveform of radial artery and its first-order and second-order time derivative.

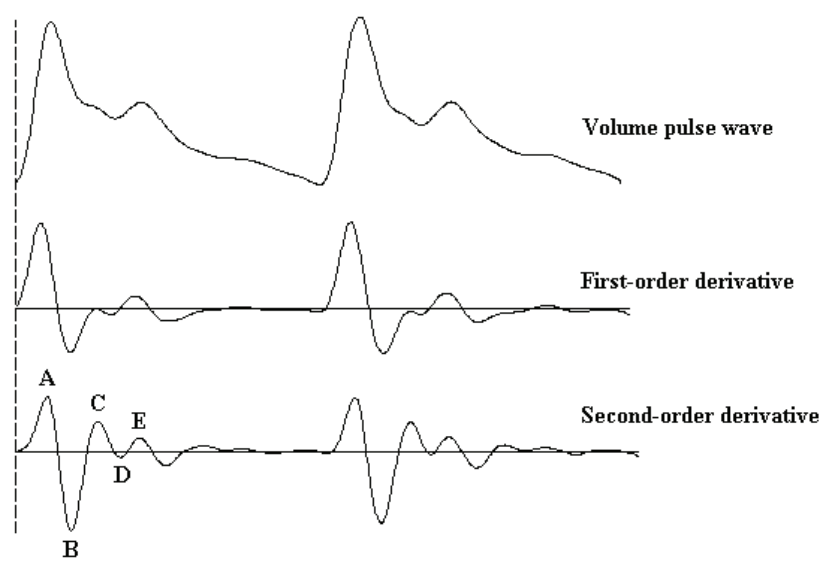

Fig. 2. Distal real volume pulse waveform and its derivatives.

The first-order derivative parameters have a nonacceptable variation and therefore are not used. However, the second-order derivative can be found frequently in literature (Hashimoto et al. 2005, Šimek et al. 2005). The abbreviations SDFAP (second derivative of finger arterial pressure waveform) or SDPTG (second derivative of finger photoplethysmograph) can also be found. The second-order derivative waveform comprised of five main parts, labeled from A to E: initial positive (A), early negative (B), re-increasing (C), late re-decreasing (D) and diastolic positive (E) (Hashimoto et al. 2005). From these determinants, the following parameters can be calculated: $\mathrm{B} / \mathrm{A}$ ratio, $\mathrm{C} / \mathrm{A}$ ration, $\mathrm{D} / \mathrm{A}$ ratio, $\mathrm{E} / \mathrm{A}$ ratio and aging index $\mathrm{AGI}=(\mathrm{B}-\mathrm{C}-\mathrm{D}-\mathrm{E}) / \mathrm{A}$. The correlations of these parameters with blood pressure and heart rate were reported. The $\mathrm{D} / \mathrm{A}$ ratio and AGI showed a positive and the $\mathrm{B} / \mathrm{A}$ ratio a negative correlation with age and $\mathrm{BP}$. The $\mathrm{D} / \mathrm{A}$ ratio and $\mathrm{AGI}$ showed a negative and the $\mathrm{B} / \mathrm{A}$ ratio a positive correlation with heart rhythm (Hashimoto et al. 2005). Parameters B/A and C/A correlated only with age. Parameter D/A correlated with age, heart period, mean blood pressure, body height and gender. Parameter E/A correlated independently with age and mean blood pressure (Šimek et al. 2005).

\section{Pulse wave velocity (PWV)}

The rate at which aortic pressure waves travel is determined in part by vascular stiffness. As large arteries stiffen with age or disease processes, both PWV and amplitude of the reflected wave increase. The reflected wave then appears in the aorta during systole, augmenting central aortic pressure.

The parameter of real spreading of pulse wave in the artery system is pulse wave velocity (PWV) or pulse transit time $(\mathrm{PTT})$, related according $\mathrm{PWV}=\mathrm{L} / \mathrm{PTT}$, where $\mathrm{L}$ is the length distance of two spots between which the velocity is measured. This of course cannot be confused with blood flow velocity, which is much slower and has a different physiological sense. PWV is the most frequently evaluated parameter, because it is very evident and measurable by commercial available devices. Pulse transit time (PTT) can be determined and referenced to the electrocardiogram R wave (Allen and Murray 2002). PWV is determined either using an automatic device, where two pressure transducers are used to record the two artery pulse waves, or using the combination of ECG Rwave and a cuff, always in the subject in supine position.

In recent studies, carotid-femoral PWV (Hashimoto et al. 2007, Waldstein et al. 2008) or brachial-ankle PWV is evaluated. Brachial-ankle PWV was significantly and positively correlated with age and 
blood pressure (Hashimoto et al. 2005). Carotid-femoral pulse wave velocity has been proposed as the gold standard for arterial stiffness measurement, because it is a more direct measure of stiffness, has good reproducibility, requires little technical expertise and there is evidence from a number of large prospective independent studies to support its use as an independent predictor of vascular outcomes (Laurent et al. 2006).

\section{Augmentation index}

Augmentation index (AIx) is defined as the difference of the first systolic and second pressure peak, expressed as a percentage of the pulse pressure. Increase of the aortic pressure will thus provide an estimate of the stiffness of the arterial system in its complexity and can be expressed numerically AIx $=(\mathrm{AP} 1-\mathrm{AP} 2) / \mathrm{AP} 1 \mathrm{x}$ $100 \%$ (Fig. 1 if measuring the pressure waveforms). AIx can be evaluated either for the aorta (central AIx, cAIx) or radial or other distal arteries (peripheral AIx, pAIx) (Hashimoto et al. 2007, Wykretowicz et. al 2007). Since AIx is influenced by heart rate, the corrected index for heart rate 75 bpm (AI@75) is commonly used (Wilkinson et al. 2000).

The measurements are provided with the automatic digital oscillometric blood pressure monitor (Omron, Omron Corporation, Kyoto, Japan) using the recommendations of the American Heart Association (Pickering et al. 2005). The short-term variation coefficient is about $7.2 \%$ (Papaioannou et al. 2007).

AIx has the strongest association with regression of LV mass in treatment-naive hypertensive subjects during one year (Hashimoto et al. 2007). Body fat content is significantly correlated with both pAIx and cAIx (Wykretowicz et al. 2007).

\section{Transformation into frequency domain (FFT)}

Using the mathematical Fast Fourier transformation (FFT) for pulse waveforms is also one of the possible approaches for evaluation, simplified by modern computers. Some of cited studies (Oliva et al. 1976, Oliva and Roztočil 1983) distinguish the healthy patients and those with arteriosclerosis on the basis of volume pulse wave FFT (measured on the toe). The fundamental frequency and lower harmonics from patient pulses were compared against normative range values. Sherebrin and Sherebrin (1990) showed how the pulse shape varied with age using 54 subjects in three age groups. The youngest group had a larger power in the second harmonic, (normalized to the fundamental) than the older two groups $(p<0.05)$. The decrease of power in the harmonics of the peripheral pulse wave with age may be a useful noninvasive measure of aging and vascular disease. Pressure recordings from 16 patients with normal pressure by hydrocephalus were analyzed during controlled intracranial hypertension by Christensen and Børgesen (1989). Single pulse pressure wave analyses were performed on a computer system with FFT. They found good correlation between the first harmonic, mean intracranial pressure, pulse amplitude, and between the fifth harmonic and rise time coefficients.

\section{Conclusions}

This review has discussed the general evaluation of pulse waveforms, either pressure or flow or volume. It has shown the potential utilization and inaccuracy of clinical measurements. Pulse waveform is a real physiological signal. The measurement is sensitive to body motion as influenced by other physiological rhythms. Despite limitations, this technology represents a promising noninvasive tool for reflecting the status of cardiovascular system both experimental and in a clinical setting. A main focus has been on the assessment of arterial elasticity. Future challenges remain in technology developments using new materials for measurement probes construction as well as standardization of obtained data for evaluating the success of therapy.

\section{Conflict of Interest}

There is no conflict of interest.

\section{Acknowledgements}

This work was supported by two grants of the Ministry of Education of the Czech Republic no. MSM 6198959216 and no. MSM 6198959215.

\section{References}

ALLEN J: Photoplethysmography and its application in clinical physiological measurement. Physiol Meas 28: R1-R39, 2007. 
ALLEN J, MURRAY A: Age-related changes in peripheral pulse timing characteristics at the ears, fingers and toes. J Hum Hypertens 16: 711-717, 2002.

BLANC VF, HAIG M, TROLI M, SAUVE B: Computerized photoplethysmography of the finger. Can J Aneasth 40: $271-278,1993$.

BROOKS B, MOLYNEAUX L, YUE DK: Augmentation of central arterial pressure in type I diabetes mellitus. Diabetes Care 22: 1722-1727, 1999.

CHALLONER AVJ: Photoelectric plethysmography for estimating cutaneous blood flow. Non-Invasive Physiological Measurements 1: 125-151, 1979.

CHEN CH, NEVO E, FETICS B, PAK PH, YIN FC, MAUGHAN WL, KASS DA: Estimation of central aortic pressure waveform by mathematical transformation of radial tonometry pressure. Circulation 95: 1827-1836, 1997.

CHRISTENSEN L, BØRGESEN SE: Single pulse pressure wave analysis by fast Fourier transformation. Neurol Res 11: 197-200, 1989.

FILIPOVSKÝ J, SVOBODOVÁ V, PECEN L: Reproducibility of radial pulse wave analysis in healthy subjects. J Hypertens 18: 1033 - 1040, 2000.

GUYTON AC, HALL JE: Textbook of Medical Physiology. Guyton $9^{\text {th }}$ edition. Saunders, Philadelphia, 1996.

HÁLEK J, KORPAS D: A device for non-invasive pulse wave measurement [Patent description], Czech Patent Office, Prague, 2003.

HASHIMOTO J, WATABE D, KIMURA A, TAKAHASHI H, OHKUBO T, TOTSUNE K, IMAI Y: Determinants of the second derivative of the finger photoplethysmogram and brachial-ankle pulse-wave velocity: the Ohasama study. Am J Hypertens 184: 477-485, 2005.

HASHIMOTO J, IMAI Y, O'ROURKE MF: Indices of pulse wave analysis are better predictors of left ventricular mass reduction than cuff pressure. Am J Hypertens 20: 378-384, 2007.

HOPE SA, ANTONIS P, ADAM D, CAMERON JD, MEREDITH IT: Arterial pulse wave velocity but not augmentation index is associated with coronary artery disease extent and severity: implications for arterial transfer function applicability. J Hypertens 25: 2105-2109, 2007.

KELLY R, HAYWARD C, AVOLIO A, O'ROURKE M: Noninvasive determination of age-related changes in the human arterial pulse. Circulation 80: 1652-1659, 1989.

KUTAL M, HÁLEK J, KORPAS D: A method measure the pulse wave using plethysmography. In: Computer-Aided Noninvasive Vascular Diagnostic. BLAZEK T (ed), Verlag Mainz, Aachen, 2003, pp 125-129.

LAURENT S, COCKCROFT J, VAN BORTEL L, BOUTOUYRIE P, GIANNATTASIO C, HAYOZ D, PANNIER B, VLACHOPOULOS C, WILKINSON I, STRUIJKER-BOUDIER H: Expert consensus document on arterial stiffness: methodological issues and clinical applications. Eur Heart J 27: 2588-2605, 2006.

MILLASSEAU SC, KELLY RP, RITTER JM, CHOWIENCZYK PJ: Determination of age-related increases in large artery stiffness by digital pulse contour analysis. Clin Sci 103: 371-377, 2002.

MILLASSEAU SC, KELLY RP, RITTER JM, CHOWIENCZYK PJ: The vascular impact of aging and vasoactive drugs: comparison of two digital volume pulse measurements. Am J Hypertens 16: 467-472, 2003.

NICHOLS WW: Clinical measurement of arterial stiffness obtained from noninvasive pressure waveforms. $A m J$ Hypertens 18: 3S-10S, 2005.

NICHOLS WW, O'ROURKE FM: McDonalds Blood Flow in Arteries. $4^{\text {th }}$ edition. Arnold, London, 1998.

OLIVA I, ROZTOČIL K: Pulse Wave in Diagnoses of Lower Limb Ischemia Disease. Avicenum, Prague, 1982.

OLIVA I, ROZTOČIL K: Toe pulse wave analysis in obliterating atherosclerosis. Angiology 34: 610-619, 1983.

OLIVA I, IPSER J, ROZTOČIL K, GUTTENBERGEROVÁ K: Fourier analysis of the pulse wave in obliterating arteriosclerosis. Vasa 5: 95-100, 1976.

O’ROURKE MF, PAUCA A, JIANG XJ: Pulse wave analysis. Br J Clin Pharmacol 51: 507-522, 2001.

PAPAIOANNOU TG, KARATZIS EN, KARATZI KN, GIALAFOS EJ, PROTOGEROU AD, STAMATELOPOULOS KS, PAPAMICHAEL CM, LEKAKIS JP, STEFANADIS CI: Hour-to-hour and week-to-week variability and reproducibility of wave reflection indices derived by aortic pulse wave analysis: implications for studies with repeated measurements. J Hypertens 25: 1678-1686, 2007. 
PEŇÁZ J: Photoelectric measurements of blood pressure, volume and flow in the finger. In: Digest 10th Int Conf Med Bio Eng, Dresden, 1973, p. 104.

PEŇÁZ J: A device for automatic non-invasive continuous measurement of blood pressure [Patent description], Czech Patent Office, Prague, 1989.

PICKERING TG, HALL JE, APPEL LJ, FALKNER BE, GRAVES J, HILL MN: Recommendations for blood pressure measurement in humans and experimental animals. Part 1: Blood pressure measurement in humans: a statement for professionals from the Subcommittee of Professional and Public Education of the American Heart Association Council on High Blood Pressure Research. Circulation 111: 697-716, 2005.

SAVAGE MT, FERRO CJ, PINDER JS: Reproducibility of derived central arterial waveforms in patients with chronic renal failure. Clin Sci 103: 59-65, 2002.

SHEREBRIN MH, SHEREBRIN RZ: Frequency analysis of the peripheral pulse wave detected in the finger with a photoplethysmograph. IEEE Trans Biomed Eng 37: 313-317, 1990.

SCHURMANN M, ZASPEL J, GRADL G: Assessment of the peripheral microcirculation isung computer-assisted venous congestion plethysmography in post-traumatic complex regional pain syndrome type I. J Vasc Res $\mathbf{3 8}$ : 453-461, 2001.

ŠIMEK J, WICHTERLE D, MELENOVSKÝ V, MALÍK J, SVAČINA S, WIDIMSKÝ J: Second derivative of the finger arterial pressure waveform: an insight into dynamics of the peripheral arterial pressure pulse. Physiol Res 54: 505-513, 2005.

TAKAZAWA K, KOBAYASHI H, SHINDO N, TANAKA N, YAMASHINA A: Relationship between radial and central arterial pulse wave and evaluation of central aortic pressure using the radial arterial pulse wave. Hypertens Res 30: 219-228, 2007.

VAITKEVICIUS PV, FLEG JL, ENGEL JH, O'CONNOR FC, WRIGHT JG, LAKATTA LE, YIN FC: Effects of age and aerobic capacity on arterial stiffness in healthy adults. Circulation 88: 1456-1462, 1993.

VLACHOPOULOS C, O'ROURKE M: Genesis of the normal and abnormal arterial pulse. Curr Probl Cardiol 25: 303-367, 2000.

WALDSTEIN SR, RICE SC, THAYER JF, NAJJAR SS, SCUTERI A, ZONDERMAN AB: Pulse pressure and pulse wave velocity are related to cognitive decline in the Baltimore Longitudinal Study of Aging. Hypertension 51: 99-104, 2008.

WILKINSON IB, MACCALLUM H, FLINT L, COCKCROFT JR, NEWBY DE, WEBB DJ: The influence of heart rate on augmentation index and central arterial pressure in humans. $J$ Physiol Lond 525: 263-270, 2000.

WYKRETOWICZ A, ADAMSKA K, GUZIK P, KRAUZE T, WYSOCKI H: Indices of vascular stiffness and wave reflection in relation to body mass index or body fat in healthy subjects. Clin Exp Pharmacol Physiol 34: 1005$1009,2007$. 